5

\section{KAWASAKI DISEASE IN TAIWAN: A 4TH NATIONWIDE SURVEY, 1994-} 2000

H C Lue ${ }^{1}$, S Philip ${ }^{1}$ Department of Pediatric Cardiology, National Taiwan University Hospital, Taipei, Taiwan ${ }^{1}$, Center for Cardiovascular Research, Department of Comparative Medicine, ATIT, Miaoli \& National Taiwan University Hospital, Taipei, Taiwan ${ }^{2}$

A Kawasaki disease (KD) survey, 4th nationwide survey was performed retrospectively to evaluate the morbidity and mortality of KD during 1994-2000 in Taiwan. This study was the continuation of past three surveillances since 1976, when the first case appeared. A specially designed questionnaire was sent to all the major hospitals, with 100 or more beds, to collect the number of patients encountered in each hospital, data with identification numbers (ID), sex, age, coronary lesions. The total number of KD cases $(1994-2000 ; n=4892)$ and incidents were also increased in this survey (year $2000=53.33$ ) with two out breaks in 1996 and 1998. Annual incidence were calculated on mid year population of less than 5 years of age. Male to female ratio was 1.7:1 and still showed constant male $(\mathrm{n}=3079)$ dominant ratio. The patients below 2, 2 to 4 and above 4 years were $57 \%$, $27 \%$ and $16 \%$ respectively. $84 \%$ cases $(n=4118)$ were below 4 years of age. Highest number of cases were reported by northern provinces $(n=2142)$ then followed by south $(n=1401)$ which was same as the previous survey. Early coronary artery dilatation was noted in 1329 cases ( $27.18 \%$ ). Fatality rate has come down to $0.06 \%(\mathrm{n}=3)$ when compared to 0.4 and $0.2 \%$ in the previous surveillance. Increasing numbers of patients with peaks, every 2-3 years suggested the possibility of unknown infectious agents playing the role in etiology. The present day advances could not decrease the prevalence rate but improved long-term outcomes significantly.

\section{6}

\section{EPIDEMIOLOGICAL ASPECTS OF KAWASAKI DISEASE IN FOUR CHI- NESE PROVINCES}

Tuohong Zhang ${ }^{1}$, Dawei Wang ${ }^{1}$, Fuyong Jiao ${ }^{1}$, Zhongdong $\mathrm{Du}^{1}$, Xiaoguang Zhou ${ }^{1}$, Hiroshi Yanagawa $^{2}$, Yosikazu Nakamura ${ }^{3}$, Tomisaku Kawasaki ${ }^{4}$ Department of Public Health, Peking University, Beijing, China ${ }^{1}$, Saitama Prefectural University, Saitama, Japan ${ }^{2}$, Department of Public Health, Jichi Medical School, Tochigi, Japan ${ }^{3}$, Kawasaki disease Research Center, Tokyo, Japan ${ }^{4}$

Objective. The objective of the study is to describe recent epidemiological patterns of Kawasaki disease in China and to make a comparison between China and Japan.Methods. The survey form and diagnostic guideline of Kawasaki disease that had been approved by the Kawasaki Disease Research Committee were sent to all pediatric departments of county hospitals and urban medical centers where pediatric beds existed in Jiangsu (197 hospitals), Shannxi (150 hospitals), Guangdong (156 hospitals) provinces and Beijing City (39 hospitals). A retrospective survey was carried out and all of the hospitalized patients during a 5-years-period had been included into this research.Results. The total hospitalized patients during a 5-years-period had been included into this research.Results. The total
number of recorded cases during the 5-years-period was 2127 . The incidence rates for per 100,000 children younger than 5 years of age were 1.85 in Jiangsu, 2.34 in Shan'xi, 24.4 in Beijing and 5.93 in Guangdong. The male:female ratio was from 1.70 to 2.23 . The peak of age at onset was 1 to 2 years old. The disease occurred more frequently in spring and summer, with the lower incidence in autumn and early winter. Patients who developed cardiac sequalae accounted for $13.6 \%, 14 \%, 21.5 \%$ and $30 \%$ in Jiangsu, Shan'xi, Beijing and Guangdong respectively. Fatality rate was $0.2 \%$
8

THE EPIDEMIOLOGY OF KAWASAKI DISEASE IN ONTARIO AND CANADA

Brian W. McCrindle ${ }^{1}$, Luella Lobo ${ }^{1}$, Seema Nagpal ${ }^{2}$, Richard Fry ${ }^{2}$, Brian Sinclair ${ }^{4}$, Frank Dicke ${ }^{3}$, Rae S.M. Yeung ${ }^{1}$ The Hospital for Sick Children, University of Toronto, Toronto, ON, Canada ${ }^{1}$, Laboratory Centre for Disease Control, Health Canada, Ottawa, ON, Canada ${ }^{2}$, Children's Hospital of Western Ontario, London, ON, Canada ${ }^{3}$, Children's Hospital of Eastern Ontario, Ottawa, ON, Canada $^{4}$

Purpose: We sought to determine recent trends in the incidence and epidemiologic features of Kawasaki disease (KD) in Ontario and Canada. Methods: A survey for incident cases from 1998-2000 was performed for all hospitals and pediatric cardiologists in Ontario. In addition, national hospital discharge abstract data from the Canadian Institute for Health Information (CIHI) was related to population denominators from census data from Statistics Canada. Results: Our previous epidemiologic survey identified an annual incidence for $1995-1997$ of 14.5 per 100,000 children aged $<5$ years in Ontario. This contrasts with an incidence of 18.9 derived from CIHI data for the same period (diagnoses not verified). From the current Ontario survey for 1998-2000, 634 incident cases were identified ( $67 \%$ males), for an annual incidence of 20.6 per 100,000 children aged $<5$ years. Cases were typical in $82 \%$ ( $50 \%$ with coronary involvement), with $7 \%$ having fewer than 5 criteria but with coronary involvement (atypical), and $11 \%$ having fewer than 5 criteria and no involvement (incomplete). Coronary aneurysms were noted in $4.1 \%$, only $54 \%$ of whom had typical KD. IVGG was given in $88 \%$, and within 10 days of fever onset in $93 \%$. Median length of stay for hospitalized cases was 2 days (range, $<1$ to 15 ), and was not related to age, number of criteria, timing of IVGG treatment or coronary complications. From CIHI data for 1994-1998 for Canada, a significant trend towards peaks in December and January, with a lesser peak in June, was noted $(\mathrm{p}<0.001)$, with no significant differences in seasonal trends between years. From CIHI data for 1979-1998, there was a significant linear increase in annual incidence $(\mathrm{p}<0.001)$ which was greater in males $(0.84$ per year) vs. females $(0.47$ per year; $\mathrm{p}<0.001)$. Conclusion: An increasing incidence is noted, which may reflect contributions of increasing physician awareness and an increased diagnosis of atypical and incomplete cases.

\section{9}

\section{EPIDEMIOLOGY OF KAWASAKI DISEASE IN A LARGE URBAN CENTER IN THE UNITED STATES OVER A 21 YEAR PERIOD}

Wilbert H. Mason, Masato Takahashi Department of Pediatrics, Childrens Hospital of Los Angeles, Los Angeles, CA, USA

Previous studies on the epidemiology of Kawasaki disease (KD) in the US have been limited to reports on epidemics or the epidemiologic characteristics of the illness in various populations over limited periods of time. Childrens Hospital Los Angeles is a 314 bed teaching hospital which serves a large urban, multi-ethnic population. We describe the epidemiology of KD in our hospital from 1979 through 2000 in a population of $980 \mathrm{KD}$ patients seen over that period. We discuss trends in incidence, age, gender, ethnicity, geographic distributon, seasonality, and birth cohort information in this group of patients. We also describe clinical characteristics including rates of recurrence, incidence of cardiovascular abnormalities, myocardial infarction, and mortality. Results of theraputic interventions are detailed especially with regard to the use of intravenous gamma globulin and steroids. In addition, information on the occurence of KD in Los Angeles County obtained from government databases for the years 1989 through 1999 is evaluated for the incidence, age, ethnicity, geographic distribution, and other epidemiologic characteristics. Trends in the two populations are compared to determine if information derived from our population is representative of that of the population as a whole. This would allow for on going surveillance of $\mathrm{KD}$ in this large urban county by reporting from a single hospital site and inferring epidemiologic trends observed to the rest of the population. Since KD is greatly under reported in Los Angeles County at present, this would improve our understanding of its epidemiology here substantially.

\section{7}

\section{KAWASAKI DISEASE IN HONG KONG, 1994-2000 SURVEILLANCE}

Yin Ming $\mathrm{Ng}^{1}$, Rita Sung ${ }^{2}$, Lok Yee $\mathrm{So}^{3}$, Nai Chung Fong ${ }^{4}$, Yan Wah Cheng ${ }^{5}$, Marco H K Ho ${ }^{1}$, Dora M L Wong ${ }^{1}$, Shuk Han Lee ${ }^{1}$, Ngai Shan Kwong ${ }^{5}$, Man Ching Yam ${ }^{2}$ Hong Kong Kawasaki Disease Study Group, Kowloon, Hong Kong, China ${ }^{1}$, Department of Paediatrics, Prince of Wales Hospital, Shatin, N. T., Hong Kong, China ${ }^{2}$, Department of Paediatrics, Pamela Youde Nethersole Eastern Hospital, Chai Wan, Hong Kong, China ${ }^{3}$, Department of Paediatrics, Princess Margaret Hospital, Kowloon, Hong Kong, China ${ }^{4}$, Department of Paediatrics, Tuen Mun Hospital, N.T., Hong Kong, China $^{5}$

Objective: The Hong Kong Kawasaki disease Study Group (HKKDSG) has started surveillance on patients diagnosed to have Kawasaki Disease (KD) in Hong Kong since 1993 with the purpose of collecting incidence data, reviewing the characteristics of KD and analyzing the epidemiological pictures of KD in Hong Kong. Methods: A retrospective survey on incidence, epidemiological data and characteristics of KD was performed. This is accomplished by asking cardiologists and paediatricians in-charge of the cases in all leading hospitals to send in the surveillance forms designed by the HKKDSG with cases diagnosed from 1 July 1994 to 30 June 2000. Results: Overall 696 cases of KD were reported with onset between 1 July 1994 and 30 June 2000. $686(98.6 \%)$ were Chinese. Their age ranged from 1 month ( 0.1 year) to 15 years ( 15.4 years) with median of 1.7 years. There were 435 boys $(62.5 \%)$ and 261 girls $(37.5 \%)$ and $620(89 \%)$ were $<5$ years of age. Infant is the largest group, comprising 209 infants ( $30 \%$ ). A total of $84.6 \%$ of children had received either $1 \mathrm{~g} / \mathrm{kg}$ (146 patients) or $2 \mathrm{~g} / \mathrm{kg}$ (443 patients) intravenous gammaglobulin. All patients were also given high dose aspirin. There were $52(7.5 \%)$ patients who had prolonged fever and some required repeated doses of intravenous gammaglobulin. The presentation was "Atypical" in $71(10.2 \%)$ children. There were only 6 recurrences noted. Coronary artery aneurysms or ectasia were present at the 4 th week in $69(10 \%)$ children. Giant aneurysms $(8 \mathrm{~mm}$ or $>8)$ was present in $3(0.4 \%)$ cases. There was no $69(10 \%)$ children. Giant aneurysms $(8 \mathrm{~mm}$ or $>8)$ was present in $3(0.4 \%)$ cases. There was no
death reported. The average yearly incidence was 32 per 100,000 children below five. Conclusion: Kawasaki disease is common in Chinese in Hong Kong with an incidence of 32 per 100,000 children below five.
10

NOVEL STAPHYLOCOCCAL SUPERANTIGENS IN CHILDREN WITH KAWASAKI DISEASE

David Burgner, Nigel Curtis, Anna Goodsall, Elena Xixi, Michael Levin Department of Paediatrics, Imperial College Faculty of Medicine, London, UK

The aetiology of Kawasaki disease (KD), particularly the role of superantigens, remains controversial. We have investigated the presence of all reported staphylococcal superantigens in KD patients and healthy controls. Methods 87 children with KD and 57 similarly aged healthy children undergoing routine vaccination were screened by bacterial nasal swabs for the presence of superantigen-producing Staphylococcus aureus. A specifically developed T-cell proliferation assay detected mitogenic activity in sterile bacterial culture supernatants, indicative of superantigen activity. The presence of the $S$. aureus-specific nuc gene and genes for all reported staphylococcal superantigen toxins (i.e. toxic shock syndrome toxin-1, staphylococcal enterotoxins (SE) A, B, C, D, E, H, K, the enterotoxin gene cluster (egc) toxins G, I, K, L and M, as well as exfoliative toxins (ExT) A and B) were detected in DNA from bacterial cultures by PCR. Results A similar proportion of KD patients and controls $(\sim 25 \%)$ had nasal colonisation with $S$. aureus. Mitogenic activity was observed in 25 $(29 \%)$ of $S$. aureus nasal isolates from KD patients versus $4(7 \%)$ of isolates from controls $(\mathrm{p}<0.005)$. S. aureus DNA encoding superantigen genes or mitogenic activity was present in $28(32 \%)$ of KD isolates, but only $9(16 \%)$ of controls $(\mathrm{p}<0.05)$. Discussion Although $S$. aureus nasal carriage is not increased in KD patients, the presence of staphylococcal superantigens, detected by both bioassay and genomic sequence analysis, strongly supports an aetiological role for superantigens in KD. Moreover these data suggest that any one of a number of different superantigen toxins, produced by $S$. aureus or possibly by other bacteria, viruses or mycoplasma, can cause KD. This might explain why efforts to find an unifying aetiological association, or a consistent $\mathrm{T}$ cell receptor $\mathrm{V} \beta$ repertoire, have so far proved unsuccessful. 